\title{
La fórmula del paraguas de soberanía ha- cia la cuestión Malvinas: ¿aplicable a la Política Exterior de Macri? ${ }^{1}$
}

\author{
The sovereignty umbrella formula in connection \\ with Malvinas: \\ Is it applicable to President Macri's foreign policy?
}

\section{Martina Mántaras²}

Resumen: Desde la redemocratización de la Argentina, la política exterior hacia la cuestión Malvinas es uno de los ejes más importantes analizados para determinar de qué manera nuestro país se proyecta hacia el mundo. Por ello, es necesario analizar cuál es la estrategia llevada a cabo por Mauricio Macri desde su llegada a la presidencia, en diciembre de 2015. Partiendo desde el diagnóstico de que el gobierno anterior se "alejó del mundo" y teniendo en cuenta que el objetivo del gobierno de "Cambiemos" es lograr una reactivación económica a partir de la búsqueda de inversiones en los países occidentales desarrollados, la hipótesis del trabajo plantea que la política exterior de Macri hacia la cuestión Malvinas se asemeja, en cierta medida, a la llevada a cabo en los años 1990 por el presidente Carlos Menem, determinada a partir de la fórmula jurídico-política llamada "paraguas de soberanía" (HERRERO, 2013: 59).

Palabras clave: Política Exterior Argentina; Paraguas de Soberanía; Gobierno de Carlos Menem; Gobierno de Mauricio Macri; Cuestión Malvinas; Dimensión Pesquera

Abstract: Since the return to democracy in Argentina, foreign policy in connection with Malvinas has been one of the most important issues to be analyzed in order to determine the way our country presents itself to the world at large. As a result, it is necessary to look into the strategies employed by Mauricio Macri since he entered office in December 2015. Based on the fact that the previous administration "pulled away from the world" and taking into consideration that the aim of "Cambiemos" is to re-activate the economy by attracting investments from developed western countries, the hypothesis formulated in this article poses that Macri's foreign policy as regards Malvinas resembles, to a certain extent, President Menem's foreign policy in the 1990, which was determined by the legal-political formula named "sovereignty umbrella" (HERRERO, 2013: 59).

DOI: https://doi.org/10.24215/23142766e063

\footnotetext{
${ }^{1}$ Artículo premiado sobre la investigación de la Cuestión Malvinas "Alfredo Bruno Bologna" de la Red Federal de Estudios sobre Malvinas ReFEM2065 CoFEI

${ }^{2}$ Estudiante de Lic. en Relaciones Internacionales, Facultad de Ciencia Política y Relaciones Internacionales, Universidad Nacional de Rosario
} 
Key words: Argentina's Foreign policy; Severeignty Umbrella; Menem Administration; Macri Administration; Malvinas Issue; Fishing

\section{Introducción}

Al hablar de la "cuestión Malvinas" en la República Argentina, nos referimos al conflicto o disputa de la soberanía entre nuestro país y el Reino Unido de Gran Bretaña e Irlanda del Norte por las Islas Malvinas, Georgias y Sándwich del Sur y los espacios marítimos circundantes (ORSO, 2011: 3). Tal como lo establece el Licenciado Javier Orso, el análisis sobre dicha cuestión se puede realizar desde diferentes aspectos, ya sea el histórico (análisis de los hechos sucedidos desde la época de la conquista y consecuente colonización de América, pasando por el comienzo de la usurpación británica a partir de 1833 y llegando a todos los reclamos realizados por Argentina desde su constitución como Estado Nación hasta la actualidad); el jurídico (el cual refiere a los documentos que avalan la integridad territorial como principio jurídico del Derecho Internacional Público); el político (cómo afecta a la Política Exterior Argentina en relación con la cuestión Malvinas, los diferentes objetivos planteados y alianzas establecidas por los diferentes gobiernos); el económico (principalmente refiere a las exploraciones y explotaciones de recursos vivos y no vivos de las Islas y espacios marítimos circundantes) (ORSO, 2011: 3 y ss.).

Por otro lado, la llegada de Mauricio Macri a la Casa Rosada conllevó un replanteo de la política llevada a cabo por el Estado argentino, de manera general, y de la Política Exterior como política pública, en particular. La nueva gestión parte del establecimiento de objetivos generales formulados por sus creencias ideológicas particulares, como lo son, en primer lugar, la inversión (tanto pública como privada, tanto nacional como extranjera) como "condición del desarrollo económico y del mejoramiento de las condiciones de vida de la población" (CORIGLIANO, 2018:68); en segundo lugar, la política exterior como política pública que funciona en pos de la atracción de capitales del exterior; y en tercer y último lugar, el rol del Estado como el ente activo que orienta las inversiones necesarias hacia las principales necesidades de carácter interno, como lo son la generación de oportunidades de empleo, obras de infraestructura y autoabastecimiento energético (CORGILIGANO, 2018, 68). Estos objetivos, basados en los postulados desarrollistas de la presidencia de Arturo Frondizi, permitieron delinear la estrategia de inserción externa que tendría la Argentina a partir de la llegada de Cambiemos a la presidencia en diciembre de 2015. Sin embargo, es necesario recalcar el diagnóstico establecido por Susana Malcorra, canciller argentina desde la llegada de Macri hasta su renuncia en junio de 2017, en una entrevista al diario "La Nación" (7 de diciembre de 2015), en la cual afirma la necesidad de "desideologizar la política exterior", al hablar sobre las políticas llevadas a cabo por su antecesora, en cuestiones referidas a Venezuela, al pago a los Holds-out y a la relación con Estados Unidos, entre otros. El pragmatismo iba a ser el eje estructurante de la PEA para, así, "empezar a hablar de relaciones exteriores como políticas de Estado" (LA NACIÓN, 2015). Esto, a su vez, se puede ver complementado por el documento denominado "Reflexiones sobre los desafíos externos de la Argentina: Seremos afuera lo que seamos adentro. Primer Documento" (firmado por personalidades argentinas importantes en generar lineamientos para la inserción de nuestro país en el mundo, como Jorge Faurie, Andrés Cisneros, Roberto Russell, Juan Battaleme, entre otros) 
de abril de 2015, en el cual se recomienda al próximo gobierno que, al establecer la estrategia de inserción argentina, se tenga en cuenta que "el destino de nuestro país, su rango internacional, su soberanía política y calidad del nivel de vida de nuestra población dependerán, en enorme medida, de la relación que consigamos establecer con el resto del mundo, partiendo de nuestra identidad occidental, como sociedad que practica y defiende las instituciones republicanas, la división de poderes, la libertad de expresión, los derechos humanos y garantías individuales" (DOCUMENTO GRUPO CONSENSO, abril 2015). Esto es importante de destacar, ya que vemos que la estrategia de inserción de Cambiemos será delineada de acuerdo a este documento: Argentina se identificará como occidental que buscará relanzar los vínculos con los países desarrollados de Occidente para cumplir los objetivos del gobierno anteriormente nombrados.

Lo anteriormente establecido es lo que nos permite justificar el por qué de la comparación con el gobierno de Carlos Menem durante la época del '90: aunque vemos que el riojano llega a la presidencia como candidato por el Partido Justicialista (el cual se caracteriza por una defensa a la producción industrial interna y al fomento del consumo del mercado interno, principalmente), los postulados principales de campaña no se tradujeron en políticas públicas relacionadas con el tinte ideológico de este partido político, sino que se establecieron políticas neoliberales, características de la época, las cuales abogaban por un achicamiento del Estado en pos del mercado y una PEA "pragmática" alineada a los intereses de los países desarrollados occidentales. Esto se evidencia en diferentes discursos tanto del presidente Menem ("seremos pragmáticos, sin hacer del pragmatismo una ideología. Seremos prácticos, sin hacer del realismo un dogma. Seremos sensatos, sin olvidar que el desarrollo es el verdadero nombre de la paz"), como de su canciller Guido Di Tella ("Argentina no está por amor con occidente sino por pragmatismo") (BOLOGNA, 1992: 74). Estas cuestiones discursivas, tanto del gobierno de Menem como del de Macri, nos permite establecer ciertos paralelismos para identificar como similares, en cierta medida, ambos puntos de partida, y poder, así comenzar a desarrollar trabajos de investigación que nos permitan analizar diferentes cuestiones y dimensiones de la PEA de la gestión "Cambiemos". Entendiendo que durante el gobierno de Menem la Política Exterior hacia la "Cuestión Malvinas" fue ampliamente conceptualizada y analizada por diferentes investigadores, es necesario comenzar a hacer lo mismo en cuanto a la PEA de Macri hacia un tema fundamental en la inserción argentina, como es el tema de la defensa de la soberanía de las Islas Malvinas, Georgias y Sandwich del Sur y sus espacios marinos circundantes.

Por lo tanto, en este trabajo nos referiremos al aspecto político de la cuestión Malvinas, estableciendo dos apartados generales de análisis, por un lado, el de la presidencia de Carlos Menem y, por el otro, el de Mauricio Macri. En ambos, se caracterizarán los contextos internacionales (tanto global como regional) en los cuales se desarrollan los gobiernos y los contextos internos de los cuales surgen, para luego analizar los objetivos de su Política Exterior y las acciones principales emprendidas hacia la cuestión Malvinas en la dimensión pesquera. De esta manera, se tratará de establecer si la aplicación de la fórmula políticojurídica del paraguas de soberanía hacia la cuestión de la soberanía de las Islas Malvinas (fórmula surgida en los Acuerdos de Madrid I y II durante la gestión menemista) es aplicable o no a la gestión de Mauricio Macri como presidente de Argentina.

Por último, la estrategia metodológica seguida en este trabajo es la analítico-descriptiva, dentro del encuadro metodológico cualitativo. Se utilizaron, principalmente, fuentes secundarias para realizar la investigación, a través de una revisión de la bibliografía sobre las diferentes 
cuestiones tratadas. También se utilizaron fuentes primarias, como comunicados de prensa, para terminar de completar la información necesaria para la investigación.

\section{Gobierno de Carlos Menem (1989-199)}

\section{i. Contexto internacional y nacional}

Carlos Saúl Menem asumió como presidente de la Argentina el 8 de julio de 1989. El nuevo dirigente debió establecer un nuevo modelo de inserción ante la incertidumbre que generó, no sólo la caída del Muro de Berlín en noviembre de 1989 y la disolución de los socialismos bajo la cortina de hierro, sino también la disolución de la URSS en 1991 (BOLOGNA, 2001: 6). Estos hechos dieron como finalizado el orden de Guerra Fría imperante desde el fin de la Segunda Guerra Mundial y el comienzo de un nuevo orden denominado "de post Guerra Fría", el cual se caracteriza por la incertidumbre y la hegemonía de los Estados Unidos. Es aquí donde tiene auge el neoliberalismo, el cual "propiciaba el achicamiento del Estado y la apuesta por el mercado como la guía de conducción más eficiente de la sociedad" (ZERO, 2012: 19) y cuyos principales intereses estaban representados por los organismos multilaterales de crédito: el Fondo Monetario Internacional (FMI) y el Grupo Banco Mundial (BM), instituciones heredadas del orden de Bretton Woods (CRUZ SOTTO, 2002: 20).

América Latina no fue ajena a este contexto: la aplicación de las recetas del Consenso de Washington (orientadas a solucionar la crisis de deuda externa de la década de 1980, además de lograr transparencia, estabilidad y crecimiento económico) es el principal ejemplo de la asimilación del neoliberalismo en la región, ya que éste establecía una reducción de las tareas del Estado (ante el fracaso del Estado de Bienestar). A su vez, a nivel regional, puede notarse el auge del modelo de integración denominado "regionalismo abierto", el cual buscaba una liberalización intragrupo, bajos niveles de protección contra terceros y una política comercial común (SANAHUJA, 2009: 14). Es importante nombrar lo anterior debido a que es una manera de demostrar la aceptación de las recetas de Washington por todos los países de América del Sur, no solamente fomentando políticas de privatizaciones y reducción del gasto público, entre otras, sino también a través de la redefinición de viejos modelos de integración regional (ALALC / ALADI, Pacto Andino) o a través de la creación de nuevos (MERCOSUR).

En cuanto a la variable doméstica, tal como se ha mencionado anteriormente, la llegada de Menem a la Casa Rosada se dio de manera precipitada, debido a la entrega de poder anticipada del presidente Raúl Alfonsín. El auge de las demandas económicas y sociales producidas por la fuga de capitales, manifestaciones, saqueos y desabastecimiento (agravado lo anterior por la hiperinflación sufrida en 1989) fue la causa de la asunción del riojano 6 meses antes de lo debido (ZERO, 2012: 20). Por lo tanto, como afirma Busso, esta "incontrovertible situación de crisis" permitió la victoria del candidato del partido opositor al alfonsinismo y lo posicionó, en la coyuntura política, como un espacio de envergadura para dar respuestas a la emergencia en la que se hallaba el país (BUSSO, 2014: 6).

\section{ii. Política Exterior Argentina en los 90}


Tanto Russell (1994) como Busso (2014) coinciden en que, los cambios operados a nivel mundial así como los problemas internos económicos y sociales condicionaron la PEA: ésta ahora estaría subordinada, junto a las demás políticas públicas, a la reforma estructural de la economía argentina, es decir, la política exterior tendría un perfil comercialista para insertarse en ese nuevo orden mundial de post guerra fría, caracterizado por la globalización, el neoliberalismo y la democracia liberal. Es por ello que, como afirma Zero, el diagnóstico del gobierno de Menem sobre ambos contextos generó una política exterior con cuatro ejes ordenadores. Éstos son el abandono de las posiciones de confrontación con los países desarrollados, el establecer un bajo perfil político en cuestiones tendientes a generar tensiones o fricciones con ellos, el reconocimiento de una posición de debilidad relativa de nuestro país con el mundo (apareciendo como conveniente la no pretensión de una posición internacional de protagonismo), y la relevancia del sistema de Naciones Unidas como el principal medio para garantizar la paz y seguridad internacionales (ZERO, 2012: 20).

Esta nueva definición de la PEA, basada en los postulados teóricos del realismo periférico de Carlos Escudé y caracterizada como "normal”, "realista" y pragmática" conllevaba una defensa del interés nacional en términos económicos y no implicaba resignar los márgenes de maniobra en los temas que afectaban los intereses económicos de nuestro país (ZERO, 2012: 21). Sí se estableció, sin embargo, que la alianza con los Estados Unidos era fundamental para la concreción de dicha política exterior comercialista; por lo tanto, el reestablecimiento o normalización de las relaciones con Gran Bretaña, principal socio de EE.UU. luego del conflicto armado de 1982 era fundamental para privilegiar una excelente relación con Occidente (Europa y EE.UU. principalmente). Esto se debe a la necesidad de inversiones extranjeras directas (principalmente compra de empresas de servicios públicos) por parte de los países de la Comunidad Económica Europea (BOLOGNA, 1992:74 y ss.). Por último, es importante establecer que el objetivo último de la PEA consistía en la incorporación de nuestro país al primer mundo, entendiendo como tal al mundo occidental liderado por EE.UU. y por la CEE (ZERO, 2012: 27).

También, es necesario recalcar que, durante el período menemista, estuvieron al frente del Ministerio de Relaciones Exteriores de nuestro país dos economistas: Domingo Cavallo y Guido Di Tella. Esto también demuestra el lugar preponderante de los aspectos económicos por encima de los políticos a la hora de establecer privilegios en la PEA (BOLOGNA, 1992: 75).

Por último, los hechos principales que confirman esta nueva orientación en la Política Exterior son la participación argentina en la fuerza multinacional encabezada por los EE.UU. en la Guerra del Golfo Pérsico, la desactivación del misil argentino Cóndor II, la posibilidad de ratificar el Tratado de Tlatelolco, el apoyo a la postura de los EE.UU. sobre el tema de Derechos Humanos en Cuba, el abandono del Movimiento de los No Alineados, el acceso a la condición de aliado extra OTAN y, en cuanto al tema que nos compete, la normalización de relaciones diplomáticas y comerciales con Gran Bretaña en los Acuerdos de Madrid I y II (BUSSO, 2014: 20). Debido a lo anteriormente nombrado, entre otras cuestiones relacionadas a los aspectos económicos de la aceptación de las recetas del Consenso de Washington, se estableció una política de alineamiento a los Estados Unidos en la década del 90 por parte del gobierno argentino, algo inédito en la historia de nuestra política exterior argentina.

\section{iii. Principales hechos hacia Malvinas}


Teniendo en cuenta, entonces, los objetivos de la PEA comercialista de esta época, la cuestión de Malvinas era clave. Como se nombró anteriormente, el objetivo último de la política exterior menemista era la incorporación al primer mundo: occidental y tradicional; por lo tanto, el reestablecer las relaciones diplomáticas con el Reino Unido era primordial, ya que así se comenzaría la "recomposición de las relaciones con Europa, dañadas por el conflicto armado por las islas del Atlántico Sur" (ZERO, 2012: 27).

Antes de analizar los hechos concretos hacia la cuestión, es importante destacar que la presidencia de Alfonsín, primer gobierno democrático luego de la dictadura, trató de discutir la cuestión sobre la soberanía de las Islas en pos de volver al estado anterior al conflicto bélico; sin embargo, la negativa de Gran Bretaña generó un cambio en la diplomacia argentina, la cual dejó de lado la instancia bilateral, para centrar sus energías en la multilateral a partir de las denuncias públicas de la ocupación de las Islas en diversos foros internacionales, como en la Asamblea General de Naciones Unidas, en la OEA y en el Movimiento de Países No Alineados (HERRERO, 2013: 59).

Esto se revierte en la época de Menem: el gobierno privilegiará la vía bilateral como estrategia de solución de conflicto sobre la multilateral (BOLOGNA, 1992: 76). El primer hecho que demuestra la concreción de esta estrategia se vio en la primera reunión entre representantes argentinos y británicos en Nueva York, en agosto de 1989, caracterizada por Bologna como "conversaciones para empezar a conversar" (BOLOGNA, 1992: 77). Como resultado, en la apertura de sesiones de la Asamblea General de Naciones Unidas de ese año, la Argentina no presenta la cuestión Malvinas, lo que constituye el abandono de una práctica continuada por nuestro país.

Sin embargo, lo más importante en este período es la firma de los Acuerdos de Madrid I y Madrid II entre la Argentina y Reino Unido: son los acuerdos que fundan la fórmula jurídico-política denominada "paraguas de soberanía". Mientras que en Madrid I (octubre 1989) se reestablecen las relaciones consulares, se reanudan las comunicaciones aéreas y marítimas, se levantan las restricciones comerciales y financieras, el gobierno británico se compromete a facilitar los vínculos de cooperación entre la Argentina y la CEE, y a reducir la zona de exclusión pesquera por parte de los británicos; en Madrid II (1990), se restablecen las relaciones diplomáticas. Es en Madrid I donde se define el paraguas, ya que los textos establecen que "nada en el desarrollo de la presente reunión (...) será interpretado como: a) un cambio de la posición de la República Argentina acerca de la soberanía o jurisdicción territorial y marítima sobre las Islas Malvinas, Georgias del Sur y Sándwich del Sur y los espacios marítimos circundantes; b) un cambio en la posición del Reino Unido acerca de la soberanía o jurisdicción sobre las Islas (...) y espacios circundantes; c) un reconocimiento o apoyo de la República Argentina o del Reino Unido acerca de la soberanía o jurisdicción territorial marítima sobre las Islas Malvinas (...) y espacios marítimos circundantes" (BOLOGNA, 1992: 77 y 78).

Una de las dimensiones en las cuales se aplica el paragua de soberanía es en la cuestión pesquera. A partir del Acuerdo de Madrid I, se constituyó un grupo de trabajo sobre pesca, el cual recién llega a una Declaración Conjunta sobre Conservación de Recursos Pesqueros en noviembre de 1990, en Londres. Dicha declaración establece que ambos gobiernos decidieron iniciar la cooperación en materia de conservación de recursos pesqueros sobre bases ad hoc, las cuales establecían que, en primer lugar, se creaba la "Comisión de Pesca del Atlántico Sur" para evaluar el estado de los recursos pesqueros, quedando tem- 
porariamente prohibida la pesca comercial por buques de cualquier bandera en el área establecida por la Declaración en su anexo (BOLOGNA, 1992: 79). Es importante esta declaración debido a que, a partir de 1987, los isleños ya habían comenzado a vender licencias pesqueras para la explotación de dichos recursos vivos, lo que dañaba el ecosistema marino de la zona al realizarse de una manera indiscriminada. A partir de esta declaración conjunta, la cual buscaba beneficios en el control y explotación conjunta de riquezas ictícolas, se logró consolidar un avance en la preservación de estos recursos, como también en otras áreas (MARTÍNEZ DEL PEZZO, 2016: 128).

Por otra parte, a lo largo de la duración del gobierno de Menem se fueron estableciendo diversos acuerdos, ya sea con Gran Bretaña o con la Unión Europea, y legislaciones internas de nuestro país, entre otros, en relación con la temática que nos compete. El primero de ellos es en 1994, cuando la Secretaría de Agricultura, Ganadería y Pesca establece el charteo ${ }^{3}$ de potero y, luego, la veda de pesca de calamares. También en este año se firma el "Acuerdo Pesquero Argentino con la Unión Europea", el cual, a partir de promover la conservación y la explotación racional de las poblaciones de peces sobre una base sostenible, conforme a las disposiciones pertinentes de la CONVEMAR, permitía la captura de 260.000 toneladas de pescado a sociedades mixtas integradas, principalmente, por españoles (LERENA, 2013: 30 y 31 y TERRIBILE, 2018: 105).

En 1995, se firma el "Acuerdo de Investigación Conjunta" entre Argentina y Gran Bretaña, el cual establecía la posibilidad de crear una comisión de investigación conjunta de los recursos pesqueros en el Mar Argentino, más allá del área de exclusión de Malvinas (LERENA, 2013: 31). Por otro lado, en 1997, se sanciona en Argentina la Ley Federal de Pesca núm. 24.922, la cual buscaba establecer un cierto orden a la cuestión de la pesca ante la evidencia de la reducción de la biomasa a niveles críticos, ya que las licencias pesqueras otorgadas para toda clase de peces, sólo era utilizada para la pesca de la merluza argentina en el año 1997; es decir, se observaba una alarmante situación de sobreexploación del caladero sudoccidental atlántico, como consecuencia de los acuerdos de pesca llevados a cabo por el Ejecutivo a espaldas del Congreso Nacional (TERRIBILE, 2018: 106). A su vez, es importante destacar que el principio rector en esta legislación es el de "la reserva del pabellón nacional", el cual ya había sido aprobado por otros países de Sudamérica, como también el establecimiento de "Cuotas Individuales Transferibles de Captura", cuestión que algunos autores establecen como ejemplo para afirmar de la profundización de la explotación del recurso pesquero (LERENA, 2013:32).

Por último, en 1999 Argentina acuerda con Gran Bretaña ciertas medidas establecidas al "combate a los busques sin licencias pesqueras" en el Atlántico Sur. Muchos autores establecen que nuestro país volvía a otorgar condiciones demasiado favorables al Reino Unido, ya que sólo pescarían buques con licencias inglesas en aguas jurisdiccionales argentinas (LERENA, 2013:33 y TERRIBILE, 2018:104). A su vez, se reconoce la condición, de manera explícita, de ribereño del actor inglés en Malvinas (TERRIBILE, 2018: 104), en concordancia con lo implementado a lo largo de la gestión Di Tella como Ministro de Relaciones Exteriores de nuestro país con la denominada "política de seducción hacia los isleños", la cual establecía reconocer a los isleños en Malvinas como una tercera parte del conflicto, para así poder llegar a encontrar una solución consensuada a la disputa con el Reino Unido (ZERO, 2012: 36).

\footnotetext{
${ }^{3}$ Se entiende por charteo el arrendamiento a casco desnudo.
} 
Es por todo lo expuesto que se afirma que, en la dimensión pesquera, se puede observar que la aplicación del "paraguas de soberanía" en la cuestión de Malvinas permitió establecer nuevos acuerdos, con actores principales del mundo occidental, sin discutir sobre la soberanía de las Islas Malvinas, Georgias y Sándwich del Sur y sus espacios marítimos circundantes.

\section{Presidencia de Mauricio Macri (2015-2018)}

\section{i. Contexto internacional y nacional}

Tal como se ha mencionado anteriormente, el orden de pos Guerra Fría se caracteriza por la incertidumbre: mientras que en la década de los 90 se establecía la idea de un sistema internacional unipolar, liderado por Estados Unidos, con la llegada del Siglo XXI, esta idea comienza a decaer, principalmente, luego de la securitización de la agenda por parte de EE.UU. a partir de los atentados del 11 de septiembre de 2001, lo que desplazó su interés y recursos a la "Guerra contra el terrorismo". Por otro lado, aparecen en la escena internacional actores emergentes, como es el caso de los BRICS (Brasil, Rusia, India, China y Sudáfrica), destacándose éstos por sus grandes mercados y la ampliación de su espacio e influencia como actores globales. Esto es importante debido a que el concepto "BRICS" se convierte, hacia el año 2011 con la incorporación de Sudáfrica al término, en un símbolo para describir el cambio de eje del poder global, desde las economías desarrolladas hacia los principales países emergentes (HAIBIN, 2012: 2). Por otro lado, la crisis financiera de 2008 también profundizó ese escenario de incertidumbre, al verse afectadas, principalmente, economías del mundo desarrollado, como la de los Estados Unidos y las de ciertos países de la Unión Europea, lo que mostró una verdadera crisis del modelo financiero basado en el pensamiento liberal norteamericano de la auto-regulación del mercado (ALAMÁ, 2009: 4).

A su vez, nuevos acontecimientos, como la salida de Gran Bretaña de la Unión Europea (BREXIT) en el 2016 y la llegada de un outsider de la política a la Casa Blanca (Donald Trump) en enero de 2017, generaron un aumento aún mayor de la incertidumbre sobre las reglas de juego en el orden internacional vigente. Esto se evidencia, principalmente, en las críticas hacia la "globalización neoliberal" por parte de aquellos países que fueron los mentores de esta concepción. Dentro de este marco, el gobierno de Trump estableció una vuelta al proteccionismo en el comercio internacional, el rechazo y revisión de los tratados de libre comercio y el evitar la relocalización en otros países de las empresas de capital norteamericano (ACTIS y BUSSO, 2017: 2), cuestiones fomentadas por los Estados Unidos desde el fin de la Segunda Guerra Mundial.

En cuanto al contexto regional, la llegada de gobiernos de izquierda o progresistas, en el comienzo del siglo XXI, puso en jaque el modelo de Estado surgido en los años noventa, debido a la gran crisis social, económica y política que éstos habían generado. Esto también se reflejó en el modelo de integración regional, ya que se comienza a cuestionar la idea de "regionalismo abierto" en cuanto a la convivencia, racionalidad, contenido y objetivos mismos de la integración en América Latina (SANAHUJA, 2009: 11). Ante ello surgen nuevos modelos de integración, identificados como "regionalismo postliberal", como es el caso del ALBA y UNASUR. Éstos se caracterizan por una preponderancia de la agenda política y de seguridad, por visiones críticas al "Consenso de Washington", por la defensa de un Estado 
neodesarrollista, por una agenda social orientada hacia la lucha contra la pobreza, desigualdad y asimetrías, por el surgimiento de políticas comunes activas en el campo de la energía, entre otros. De esta manera, se demuestra una caída de la relevancia de la agenda comercial, característica primordial del modelo de integración regional de la década del 90 (SANAHUJA, 2009: 44).

Sin embargo, a partir de 2011 se habla del deterioro del "regionalismo postliberal" ante la aparición en escena de la Alianza del Pacífico, modelo creado por Chile, Colombia, México y Perú (países que se caracterizaron en la etapa de "regionalismo postliberal" por no haber dado ese "giro hacia la izquierda" o progresista y por haber continuado con una política de alineamiento con los Estados Unidos). Este modelo, basado en los principios teóricos de Baldwin de "regionalismo del siglo XXI", busca congeniar "el vacío entre las normas del Siglo XX y las necesidades del comercio del Siglo XXI" (ACTIS y BUSSO, 2017: 57). Con la llegada de Mauricio Macri en 2015 y de Michel Temer en 2016 a las presidencias de Argentina y Brasil, respectivamente, se comienza a buscar una participación de estos países en dicha Alianza del Pacífico, consagrándose el deterioro de los modelos de integración postliberales. Sin embargo, el Brexit y la llegada de Trump, anteriormente descripta en este trabajo, conllevan una crisis del modelo globalizado neoliberal, deteriorando así el modelo de inserción planteado por los nuevos, y otros no tan nuevos, gobiernos liberales en América Latina: los países desarrollados están en la senda de abandono del multilateralismo, de la cooperación institucionalizada y de la misma idea de integración regional, para rescatar conceptos de proteccionismo y nacionalismo, en diferentes dimensiones (ACTIS y BUSSO, 2017: 58).

En cuanto al contexto interno, la Alianza Cambiemos (PRO-UCR-Coalición Cívica) se vio como alternativa al modelo kirchnerista imperante desde el 2003 (Presidencia de Néstor Kirchner 2003-2007 y las dos presidencias consecutivas de Cristina Fernández 2007-2015). Las causas del triunfo de la oposición al gobierno peronista se puede ver a partir de su agotamiento: hechos como el asesinato del fiscal Alberto Nisman en enero de 2015, la insostenible situación económica del país (el impacto de la baja del precio internacional de los commodities, una inflación del 30\%, la caída de las reservas del Banco Central) y las denuncias de corrupción fueron algunas de las principales cuestiones que explican la victoria de Mauricio Macri en el ballotage de noviembre de 2015 (MURILLO, RUBIO Y MANGONNET, 2016: 1).

\section{ii. Política Exterior Argentina}

Ante el panorama internacional y contexto interno descriptos, Mauricio Macri plantea una política exterior diferente a la de sus antecesores. Mientras los gobiernos kirchneristas planteaban un diagnóstico de relativa declinación del mundo occidental desarrollado (Estados Unidos y Europa Occidental) y de relativo ascenso de nuevos polos de poder como China, fundamentalmente, y se establecía así una política de diversificación de vínculos en diferentes dimensiones (política y económica-comercial) (CORIGLIANO, 2018:63, y MORGENFELD, 2017:1), la gestión de Cambiemos establece un cambio en la orientación internacional de nuestra política exterior (HERMANN, 1999: 5). Este cambio no sólo se debe a cuestiones de carácter ideológico, tal como establece Corigliano (2018) al afirmar que "las creencias filosóficas parten de una visión menos hostil que la de su antecesora respecto del proceso de globalización liberal (...) [en cuanto] a los posibles beneficios (...) para el desarrollo económico y la generación de empleo interno en la Argentina, [lo cual] está, a su vez, acom- 
pañado por la creencia instrumental macrista de la necesidad de promover el multilateralismo económico y político y las reglas de gobernabilidad económica y política global en los distintos foros internacionales (G-20, OMC, Naciones Unidas), como escudos protectores"; sino también a cuestiones referidas a cambios sistémicos desde antes de la finalización del gobierno de Cristina Fernández, como por ejemplo por la caída del precio de los commodities, generando que grupos de la oposición política y grupos empresariales empezaran a ejercer presión por un cambio en la manera de insertarse la Argentina en el mundo (BUSSO y ZELICOVICH, 2016:17).

Por lo tanto, la PEA en el gobierno de Macri se va a caracterizar por un perfil occidental que privilegia los vínculos con EE.UU. y Europa (BUSSO y ZELICOVICH, 2017:18) y es concebida como un "instrumento de promoción del desarrollo económico argentino" (CORIGLIANO, 2018:65). En este marco, acciones orientadas hacia la participación argentina en la Alianza del Pacífico, la flexibilización del Mercosur, el pago de los Holds-out, la participación en el Foro Económico de Davos, la visita del presidente Barack Obama, entre otras, evidencian ese cambio de orientación de nuestra Política Exterior.

Por último, tanto Busso y Actis (2017) como Corigliano (2018) coinciden en que esta estrategia de inserción va a ser truncada o más prudente con la aparición de Donald Trump en la escena internacional, ya que mientras que con Barack Obama se compartía una visión liberal y multilateralista del mundo, con reglas claras definidas; Donald Trump promueve políticas de proteccionismo comercial y nacionalismo, que no coinciden con los lineamientos de la PEA formulada por Macri y su equipo, a partir de enero de 2017 (CORIGLIANO, 2018: 64).

\section{iii. Principales hechos hacia Malvinas}

Teniendo en cuenta esta Política Exterior de Macri orientada hacia Occidente, Gran Bretaña no escapa del diagrama establecido por Cancillería como socio comercial posible. Es por ello que, a partir de diciembre de 2015, se establece por un lado un relanzamiento del vínculo bilateral y, por otro, que dicho relanzamiento no sea truncado por la disputa sobre la soberanía de las Islas Malvinas, en especial en el (esperado) avance del comercio y la inversión (CASTAÑO, 2017: 8). Esto se establece a partir de la crítica de la gestión Cambiemos hacia la política llevada a cabo por los gobiernos kirchneristas (principalmente de Cristina Fernández) hacia la cuestión Malvinas, la cual se caracterizó por una defensa en los foros multilaterales, por una defensa a los recursos naturales y por la cooperación en cuestiones humanitarias. Algunos analistas la clasificaron como una política de confrontación, debido a que existieron ciertos cortocircuitos en la relación con Gran Bretaña y los isleños ${ }^{4}$; sin embargo, tal como lo estipula la Constitución Argentina a partir de la reforma de 1994, con los gobiernos kirchneristas la cuestión Malvinas se situó "en lo más alto de la política exterior argentina" (HERRERO, 2013: 63).

Es por ello que, tal como se venía planteando en el trabajo, podemos ver también un cambio de orientación de la PEA hacia la cuestión Malvinas, con una reducción en la intensidad del reclamo por la soberanía de las Islas Malvinas, Georgias y Sandwich del Sur, y sus espacios marinos circuncidantes. Al igual que en los años 90, observamos una subordinación del reclamo sobre Malvinas a los objetivos económico-comerciales del gobierno de Mauricio

\footnotetext{
${ }^{4}$ véase Herrero, Luciano: "La cuestión de Malvinas en la Política Exterior Kirchnerista".
} 
Macri. Esto se evidencia, en primer lugar, en el comunicado conjunto argentino-británico de septiembre de 2016 del vicecanciller argentino de ese momento, Carlos Foradori, y el Ministro de Estado para Europa y las Américas de la Secretaría de Relaciones Exteriores y Commonwealth británica, Sir Alan Duncan (llamado de manera coloquial Acuerdo Foradori-Duncan). En este acuerdo, se establece que "la reunión bilateral sirvió para que ambos funcionarios pudieran continuar impulsando una agenda omnicomprensiva, multidimensional y multisectorial, basada en la complementariedad entre ambas naciones, orientada al fortalecimiento de la relación bilateral y a la búsqueda de soluciones a los problemas globales dentro de los marcos multilaterales que correspondan" (COMUNICADO DE PRENSA, 2016). Luego, se enumeran las cuestiones de agenda importantes para la relación bilateral: la reanudación de Consultas de Alto Nivel, las medidas en pos de la lucha contra la corrupción y el crimen organizado, políticas hacia el arte, la cultura, la educación y los deportes, medidas para incrementar el comercio y las inversiones bilaterales, medidas en materia de cooperación en el marco del G-20, el apoyo de Gran Bretaña a la incorporación de Argentina a la OCDE, cooperación en materia de Seguridad Internacional y Defensa, el apoyo británico a las medidas argentinas en materia de la crisis de refugiados imperante y, por último, cuestiones referidas al Atlántico Sur. En este último apartado, nunca se nombra la cuestión Malvinas, y se recupera la fórmula jurídico-política del paraguas de soberanía al establecer que "ambos gobiernos acordaron que la fórmula de soberanía del párrafo 2 de la Declaración Conjunta del 19 de octubre de 1989 se aplica a este Comunicado Conjunto y a todas sus consecuencias". Sólo se establece que se acuerda "adoptar las medidas apropiadas para remover todos los obstáculos que limitan el crecimiento económico y el desarrollo sustentable de las Islas Malvinas, incluyendo el comercio, pesca, navegación e hidrocarburos". A partir de allí, se contempla la eliminación de medidas restrictivas en el área de petróleo, gas y pesca en torno a Malvinas, cuestiones referidas a la navegación, comercio y vuelos sobre las islas, y el apoyo de ambas partes al proceso de identificación de ADN con relación a los soldados argentinos no identificados sepultados en el cementerio de Darwin.

En este sentido, se ve la pérdida de centralidad de la cuestión Malvinas, pero no el abandono del reclamo de soberanía, según expresiones de Susana Malcorra, canciller argentina en este período (CORIGLIANO, 2018: 79).

Luego, pocos días después del comunicado y en el ámbito de la apertura de sesiones de la Asamblea General de Naciones Unidas, se da una reunión entre la Primera Ministra británica, Theresa May, y el presidente argentino Mauricio Macri, luego de la cual el presidente declaró a los medios de comunicación que May estaba dispuesta a "negociar sobre la soberanía de Malvinas". Esto fue totalmente negado en un comunicado del Foreign Affairs y generó críticas en gran parte de la sociedad civil, militantes políticos y comunicadores en Argentina. Según Corigliano (2018): "a partir de ese momento las conversaciones entre Buenos Aires y Londres ingresaron en una etapa de impasse, evidenciando el peso de la cuestión soberanía en Malvinas como un obstáculo en el diálogo bilateral".

Todos estos hechos se enmarcan en pos de avanzar en temas de comercio e inversiones a partir de la salida de Gran Bretaña de la Unión Europea (BREXIT), proceso que se inició a partir del referéndum del 2016. Como afirma Corigliano (2018): “el corrimiento de la centralidad, aunque no necesariamente renuncia, del reclamo de la cuestión de la soberanía en las Malvinas como moneda de cambio de la intensificación de los lazos comerciales y de inversiones entre Londres y Buenos Aires, (...) [fue el tratar de aprovechar] en favor de la Argentina la salida británica de la Unión Europea (...) y el renovado interés británico por 
invertir en el mercado local en áreas como servicios y minería en el contexto de redefinición de los lazos económicos entre Londres y la UE".

Al hablar sobre la dimensión pesquera, además del acuerdo Fioradori-Duncan, sólo se puede observar que en mayo de 2018, en el marco de la 41a Reunión del Tratado Antártico (celebrada en Buenos Aires), el Subcomité Científico de la Comisión de Pesca del Atlántico Sur mantuvo reuniones de dos días entre funcionarios científicos de Argentina, Gran Bretaña y del gobierno de ocupación de las Islas Malvinas (establecida por la Declaración Conjunta del 28 de noviembre de 1990). Este Subcomité Científico no se reunía desde 2004, año en el que el presidente Néstor Kirchner discontinuó su funcionamiento. Todas las partes consensuaron la reanudación en materia de tareas de investigación conjunta sobre especies compartidas (REVISTA PUERTO, 13 de mayo de 2018). A su vez, en el comunicado de prensa de Cancillería Argentina vuelve a nombrar que dicha reanudación de actividades del Subcomité Científico se llevó a cabo bajo la fórmula de soberanía establecida en la Declaración Conjunta de Madrid de 1989, es decir, bajo la fórmula del paraguas. Por otro lado, este hecho generó críticas desde la provincia de Tierra del Fuego (ente administrativo de las Islas Malvinas) por no ser incluida en la reunión (REVISTA PUERTO, 13 de mayo de 2018).

En consecuencia, podemos observar ciertas coincidencias en la PEA en relación con Malvinas entre el gobierno de Carlos Menem, desarrollado en la década del 90, y en la gestión de Mauricio Macri, hasta septiembre de 2018.

\section{Conclusión}

Para concluir, de acuerdo a lo expuesto en este trabajo, podemos afirmar que hoy en día volvió a ser aplicada la fórmula jurídico-política del "paraguas de soberanía" en la cuestión Malvinas por parte del gobierno de Macri (como se aplicó en los '90 durante la gestión menemista), tal como se expresa en los diferentes comunicados de Cancillería. Ante una crítica a la política de "remalvinización" llevada a cabo por los gobiernos kirchneristas y ante el objetivo de atraer inversiones británicas a nuestro país para lograr la "reactivación económica", Cambiemos decidió bajar el perfil en el reclamo de la soberanía y lograr acuerdos diversos en otras áreas de cooperación para así generar "confianza" en los inversores extranjeros británicos. A su vez, vemos que se relanzaron programas de cooperación en materia de recursos naturales vivos y no vivos, como recursos ictícolas y los hidrocarburos, los cuales habían sido congelados durante el kirchnerismo. Esto se justificó a partir del reclamo en relacion con el cumplimiento de la Resolución 2065 de la Asamblea General de Naciones Unidas (principal logro diplomático argentino con relación al conflicto a partir de la vía multilateral, logrado en 1965 durante la presidencia de Arturo Illia), la cual establece el llamado a las partes en litigio a sentarse a negociar ante el problema de soberanía imperante por el territorio de las Islas Malvinas, Georgias y Sandiwch del Sur y sus espacios marítimos circundantes.

Por otro lado, no sólo vemos una coincidencia del diagnóstico sobre la situación económica argentina entre los dos gobiernos, sino también las estrategias de inserción internacional de nuestro país en el mundo subordinadas a objetivos solamente económicos planteados por ambos gobiernos analizados. A su vez, observamos un contexto regional cercano a una política de alineamiento hacia los Estados Unidos, en donde Argentina aparece como uno de los protagonistas en el subcontinente sudamericano para llevar a cabo esta política. Mientras que en la década de 1990 se estableció el Mercosur como principal modelo de 
integración regional en pos de la liberalización de las barreras que entorpecieran el libre comercio entre Argentina, Brasil, Paraguay y Chile; la llegada de Macri significó un viraje en materia de integración, en cuanto a lo que se venía dando desde comienzos del Siglo XXI con la llegada y consolidación de los gobiernos "progresistas" en la región latinoamericana. En este sentido, el actual presidente argentino apostó por una flexibilización en el Mercosur (en pos de la firma del acuerdo de libre comercio con la Unión Europea) y por un giro hacia el Pacífico, al demostrar querer pertenecer a la Alianza del Pacífico, constituida en 2011 por aquellos países latinoamericanos con acuerdos de libre comercio con Estados Unidos.

Sin embargo, a nivel global, vemos que los contextos internacionales en los cuales se debe insertar la Argentina son bastantes diferentes. Mientras que en la década de los noventa, los Estados Unidos establecieron las reglas de comportamiento a nivel global, al ser el gran ganador de la Guerra Fría; la llegada de Donald Trump a la Casa Blanca generó un clima de aún mayor incertidumbre ante medidas proteccionistas y nacionalistas en materia económica-comercial, diferente de lo que se venía dando desde la presidencia de Bush padre en la pos Guerra Fría, caracterizada por una defensa al libre comercio y relocalización de las empresas hacia el exterior. Ante este panorama, la estrategia de inserción del Gobierno de Cambiemos se vio truncada, situación bastante diferente a la del gobierno de Menem, momento en el cual la Argentina fue calificada como la "mejor alumna" en aplicar las recetas neoliberales emanadas por el Consenso de Washington.

Por último, en cuanto al tratamiento de la dimensión pesquera en la PEA hacia Malvinas, vemos una diferencia sustancial entre las dos gestiones: en el gobierno de Menem vemos muchas acciones en pos de generar una cooperación bilateral consolidada en la cuestión, en pos de generar ciertos beneficios de tipo económico; en cambio, en el período del gobierno de Macri, hubo solamente un pequeño avance, producido en mayo del corriente año, al relanzar el Subcomité Científico de Pesca. Esto puede encontrar explicación en los cortocircuitos que se dieron en la relación bilateral entre Argentina y Gran Bretaña (ante los dichos de Macri en el marco de la inauguración de la Asamblea General de Naciones Unidas en 2016), por un desinterés en la relación bilateral por parte del Reino Unido ante el fenómeno del Brexit, y la llegada de Trump y sus cambios en las reglas de juego en el orden internacional.

\section{Bibliografía}

ACTIS, Esteban y BUSSO, Anabella. (2017). “Globalización “descarriada” y "regionalismo desconcertado" en la era Trump", en RAIGAL: Revista Interdisciplinaria de Ciencias Sociales, Villa María, Córdoba, n.3, pp. 51-64.

ALAMÁ, Luisa y FUERTES EUGENIO, Ana María. (2009). “Cronología de la crisis económica y debilidades de la globalización: la economía española en el contexto internacional", Revista de Treball: Economía y Sociedad, Barcelona, n. 53, pp. 9-17.

BOLOGNA, A. Bruno. (1992). "El conflicto de las Islas Malvinas”, Rosario, Ediciones Facultad, pp. 25-87.

BOLOGNA, A. Bruno. (2001). "Nuevas percepciones del sistema internacional en la posguerra fría”, en Cuadernos de Política Exterior Argentina, Rosario, Serie Docencia № 64, CERIR. 
BUSSO, Anabella. (2014). "Los vaivenes de la política exterior argentina re-democratizada (1983-2013). Reflexiones sobre el impacto de los condicionantes internos", en Estudios Internacionales, Universidad de Chile, Santiago de Chile, v.46 n.177, pp. 9-23.

BUSSO, Anabella y ZELICOVICH, Julieta. (2016). "El gobierno de Mauricio Macri y la Integración Regional: ¿desde el Mercosur hacia la Alianza del Pacífico?”, Revista Conjuntura Austral, Porto Alegre, Brasil, v.7, n.37, pp. 17-24.

CASTAÑO, Francisco. (2017). "La inserción internacional argentina durante el gobierno de Macri", Ponencia dictada en el marco del VIII Encuentro del CERPI y VI Jornada del CENSUD, La Plata.

COMUNICADO DE PRENSA DE CANCILLERÍA, 13/09/ 2016, Buenos Aires, Argentina. https://www.cancilleria.gob.ar/es/actualidad/comunicados/comunicado-conjunto-9

COMUNICADO DE PRENSA DE CANCILLERÍA, 09/05/2018, Buenos Aires, Argentina. https://www.cancilleria.gob.ar/es/actualidad/comunicados/reunion-del-subcomite-cientifico-de-la-comision-de-pesca-del-atlantico-sur

CORIGLIANO, Francisco. (2018). "Flexibilidad en un mundo incierto: Creencias, espacios y lineamientos de la política exterior del gobierno de Macri al promediar el mandato", Perspectivas Revista de Ciencias Sociales, Rosario, a.3, n.5, pp. 62-97.

CRUZ SOTO, Luis Antonio. (2002). “Neoliberalismo y globalización económica. Algunos elementos de análisis para precisar los conceptos", Contaduría y Administración, Universidad Nacional Autónoma de México Distrito Federal, México, núm. 205, pp. 13-26.

DOCUMENTO DEL GRUPO CONSENSO. 2015. "Reflexiones sobre los desafíos externos de la Argentina: Seremos afuera lo que seamos adentro. Primer Documento".

HAIBIN, Niu. (2012). "Los BRICS en la Gobernanza Global, ¿una fuerza progresista?”, en Dialogue on Globalization, Friedrich Ebert Stiftung (FES), Nueva York, pp. 1-8.

HERMANN, Charles, F. (1999). "Changing course: When Governments choose to Redirect Foreign Policy", International Studies Quarterly, v. 34, n. 1, pp. 3-21.

HERRERO, Luciano. (2013). “La 'Cuestión Malvinas' en la política exterior durante los últimos 10 años (2003- 2013)", Contexto Internacional, FUNIF, Rosario, n. 36, pp. 5866.

LA NACIÓN, 07/12/2015, Buenos Aires, Argentina.

LERENA, César Augusto. (2013). "Política Pesquera para la Soberanía en el Atlántico Sur y Malvinas", Publicaciones de la Fundación "Nuestro Mar", Buenos Aires.

MARTíNEZ DEL PEZZO, Alberto. (2016). "Malvinas. Política exterior argentina (1989-2015)", Centro de Estudios Avanzados, Universidad Nacional de Córdoba, Córdoba, n³6, pp. 121-140.

MORGENFELD, Leonardo. (2017). “Macri y el fracaso de la subordinación a Estados Unidos: 
de Obama a Trump", Revista Realidad Económica, Buenos Aires, pp. 1-4.

MURILLO, Ma. Victoria, RUBIO, Julia María y MANGONNET, Jorge. (2016). “Argentina: El protagonismo de los votantes y la alternancia electoral", Revista de Ciencia Política, Santiago de Chile, v.36, n.1, pp. 3-26.

SANAHUJA, José Antonio. (2009). "Del "regionalismo abierto" al "regionalismo post-liberal”. Crisis y cambio en la integración regional en América Latina", Anuario de la integración de América Latina y el Gran Caribe 2008-2009. Coordinadora Regional de Investigaciones Económicas y Sociales (CRIES), pp. 11-54.

SANAHUJA, José Antonio. (2017). “Regionalismo e integración en América Latina: de la fractura Atlántico Pacífico a los retos de una globalización en crisis", en Pensamiento Propio no21, Universidad Complutense de Madrid, Madrid, pp. 29-76.

ORSO, Javier. (2011). "La distinción entre cuestión y problemas sobre Malvinas como herramienta para la comprensión de la política exterior argentina", Boletín № 15, Año 5, Departamento de Islas Malvinas, Antártida e Islas del Atlántico Sur, Instituto de Relaciones Internacionales, Universidad Nacional de La Plata, La Plata, pp. 1-20.

REVISTA PUERTO, 13/08/2018, Buenos Aires, Argentina.

RUSSELL, Roberto. (1994). "Los ejes estructurantes de la política exterior argentina: apuntes para un debate", Facultad Latinoamericana de Ciencias Sociales (FLACSO), Programa Argentina, Serie de Documentos e informes de Investigación, Buenos Aires, n.158, pp. 1-22.

TERRIBILE, Horacio A. (2018). “La política pesquera argentina. Las cuestiones de la política doméstica y el sistema internacional. Desde Malvinas al final del siglo", Boletín del Centro Naval 847, Buenos Aires, pp. 96-111.

ZERO, Lara. (2012). "La política de seducción en la cuestión Malvinas durante los gobiernos de Carlos Saúl Menem: bajo la gestión del Canciller Guido Di Tella (1991/1999)", Tesis de grado, Facultad de Ciencia Política y Relaciones Internacionales, Universidad Nacional de Rosario, Rosario, pp. 19-47 